\title{
Roads to new guanidine chemistry from 2-imidazolidinones through 2-chloroamidinium derivatives
}

\author{
Tsutomu Ishikawa \\ Graduate School of Pharmaceutical Sciences, Chiba University, 1-33 Yayoi, Inage, Chiba \\ 263-8522, Japan \\ E-mail: benti@p.chiba-u.ac.jp
}

\begin{abstract}
New guanidine chemistry from 2-imidazolidinones through 2-chloroamidinium derivatives is introduced. At first the synthetic utility of 2-cloro-1,3-dimethylimidazolinium chloride (DMC), derived from 1,3-dimethylimidazolidinone, mainly focusing on dehydration, is discussed. Modified guanidines and guanidinium salts can be prepared from DMC and its analogs by aminations with primary and secondary amines, respectively. The roles of modified guanidines not only chiral auxiliaries in asymmetric synthesis but also the nitrogen source of aziridine skeletons in guanidinium ylide-participating aziridine synthesis are discussed.
\end{abstract}

Keywords: Asymmetric synthesis, aziridine, chiral auxiliary, chloroamidininium salt, dehydration, guanidine, guanidinium ylide, 2-imidazolidinone, organic catalyst, reaction mechanism

\section{Contents}

1. Introduction

2. DMC (2) as a powerful dehydrating agent

2.1 Intermolecular condensation (Acylation)

2.2 Intramolecular dehydration (Cyclodehydration)

2.3 Chlorination

2.4 Oxidation

2.5 Reduction

2.6 Rearrangement

3. Modified guanidines as chiral auxiliaries 


\subsection{Preparation}

3.2 Roles as chiral auxiliaries

3.3 Application to asymmetric synthesis

4. Guanidinium ylides for aziridine formation

4.1 Reaction using achiral guanidinium ylides

4.2 Application to asymmetric synthesis

4.3 Mechanistic approaches

4.4 Proposed mechanisms

5. Conclusions

\section{Introduction}

Relatively stable urea function, $\mathrm{R}_{2} \mathrm{NCONR}_{2}$, can be functionalized to more reactive species available in organic synthesis. 2-Imidazolidinones are representative cyclic ureas, among which 1,3-dimethyl-2-imidazolidinone (DMI) (1) is widely used in industrial scale as non-toxic and aprotic polar solvent. Dehydration reaction is one of the most fundamental and important reactions in organic synthesis and, thus, lots of versatile reagents usable under mild conditions, such as dicyclohexylcarbodiimide (DCC), have been explored until now. Chlorination of $\mathbf{1}$ affords 2-chloro-1,3-dimethylimidazolinium chloride (2) with chloroamidinium function, which can act as an alternative powerful dehydrating agent under nearly neutral conditions and now appears in "The Merck Index (13 ${ }^{\text {th }}$ ed.)" as DMC (No. 3234 in p 596). On the other hand guanidines can be classified as the strongest organic bases due to the effective resonance stabilization of their conjugated acids. We found that the chlorine atom of $\mathbf{2}$ is easily displaced with various primary amines to give the corresponding guanidine derivatives 3 , which could participate to asymmetric synthesis as potential chiral auxiliaries after the introduction of chirality into 3 , and that either alkylation of $\mathbf{3}$ or direct amination of $\mathbf{2}$ with secondary amines provide the corresponding guanidinium salts $\mathbf{4}$, which could be transformed to hitherto unknown guanidinium ylides by treatment with base. Here, we present roads to new guanidine chemistry from 2-imidazolidinones through 2-chloroamidinium derivatives.

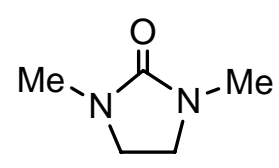

1

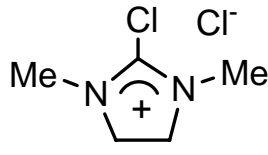

2

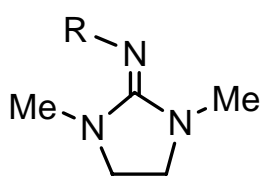

3

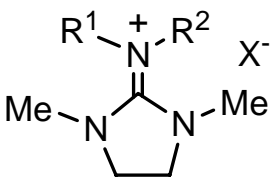

4 


\section{DMC (2) as a powerful dehydrating agent}

Chloroamidinium salts such as DMC (2) and $N, N, N^{\prime}, N^{\prime}$-tetramethylchloroformamidinium chloride have been used in dehydration reactions; however, much attention has never been paid to these reagents until now in spite of their potential ability to act as dehydrating agents. $\mathrm{We}^{1}$ focus on 2 and its synthetic utilities have been systematically studied because of its dual advantages of simple preparation from the corresponding cyclic urea, DMI (1), by chlorination (Scheme 1) and the easy removal of 1 regenerated after the condensation reaction by washing with water. DMC (2), colorless and odorless prisms, mp $95-100^{\circ} \mathrm{C}$ (decomp.), is prepared by treatment of $\mathbf{1}$ with oxalyl chloride or trichloromethyl chloroformate (diphosgene) in place of phosgene originally used in the chlorination. ${ }^{2}$ Although $\mathbf{2}$ is stable to oxygen, moisture causes it to gradually decompose to $\mathbf{1}$ and hydrochloric acid.

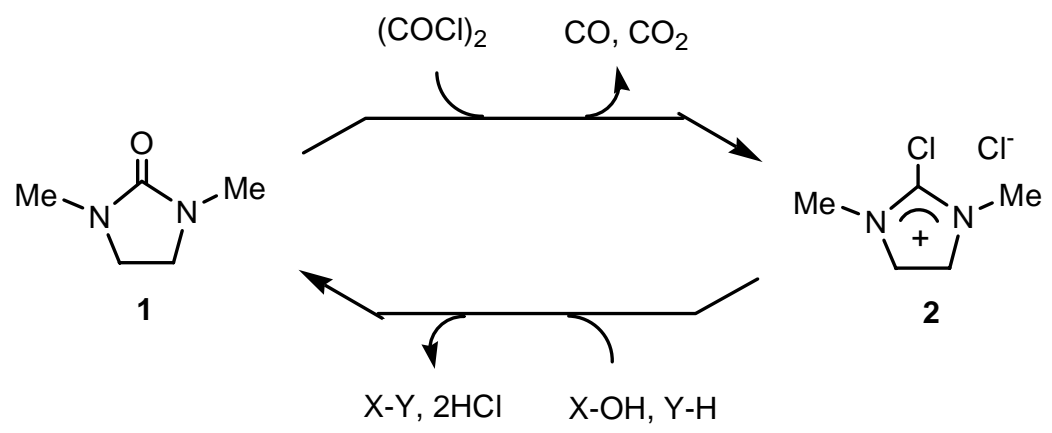

Scheme 1. Chemical relation between DMI (1) and DMC (2)

\subsection{Intermolecular condensation (Acylation) ${ }^{1 \mathrm{a}}$}

DMC (2) was first reported in 1972 in the amidation of penicillanic acid. ${ }^{2}$ We also showed that 2 acts as an effective dehydrating agent in intermolecular condensations between carboxylic acids and a variety of nucleophiles containing an active hydrogen.

In a typical experiment, a carboxylic acid (1 eq.), a nucleophile ( 1 eq.), and 2 (1 eq.) in dichloromethane are treated with pyridine ( 2 eq. $)$ at room temperature and then crude products are isolated by a conventional two-phase partition method. This method is applicable to a variety of esterification containing hindered components such as $t$-butanol or pivalic acid. Selected examples are given in Table 1 .

The acylation of 2-mercapto-1,3-thiazoline and cyclic 1,3-diones can also be carried out and in the latter case $O$ - and $C$-acylated products are obtained as a mixture dependent upon the reaction conditions used. 
Table 1. DMC-mediated esterifications

\begin{tabular}{|c|c|c|c|c|c|}
\hline \multirow{2}{*}{\multicolumn{2}{|c|}{$\begin{array}{c}\mathrm{R}^{1} \mathrm{CO}_{2} \mathrm{H} \\
(1 \text { eq.) }\end{array}$}} & \multirow{2}{*}{$\begin{array}{l}\mathrm{R}^{2} \mathrm{OH} \\
(1 \text { eq.) }\end{array}$} & \multicolumn{2}{|r|}{$\begin{array}{l}\text { pyridine } \\
\text { ( } 2 \text { eq.) }\end{array}$} & \multirow{2}{*}{$\mathrm{R}^{1} \mathrm{CO}_{2} \mathrm{R}^{2}$} \\
\hline & & & $\mathrm{CH}_{2} \mathrm{Cl}_{2}$ & $\mathrm{rt}$ & \\
\hline runs & & & $\mathrm{R}^{2}$ & time $(\mathrm{h})$ & yields $(\%)^{a}$ \\
\hline 1 & & $\left.\mathrm{H}_{2}\right)_{4}$ & $\mathrm{PhCH}_{2}$ & 2 & 96 \\
\hline 2 & & & Et & 2 & 95 \\
\hline 3 & & $\left(\mathrm{CH}_{2}\right)_{5}$ & 4- $\left(\mathrm{EtO}_{2} \mathrm{C}\right) \mathrm{Ph}$ & 12 & 76 \\
\hline 4 & & & $\mathrm{Ph}\left(\mathrm{CH}_{2}\right)_{2}$ & 20 & 91 \\
\hline 5 & & $\left.f_{2}\right)_{2}$ & ${ }^{\mathrm{t}} \mathrm{Bu}$ & 24 & 88 \\
\hline
\end{tabular}

${ }^{a}$ Non optimized, isolated yield

\subsection{Intramolecular dehydration (Cyclodehydration) ${ }^{1 \mathrm{~b}}$}

DMC (2) is also effective in intramolecular dehydration reactions. Nitriles are obtained from oximes and carboxamides. Isocyanides, isothiocyanates, and carbodiimides are similarly prepared from formamides, dithiocarbamates, and thioureas, respectively. 2 can be also applied to the construction of heterocycles based on dehydration reactions. Thus, azetidin-2-ones ( $\beta$-lactams) are prepared in good yields by treatment of carboxylic acids with imines in the presence of 2 and triethylamine. (Table 2) The stereochemistry of major products is controlled by a substituent on carboxylic acids used.

Cyclodehydration of $\gamma$-keto acid derivatives with 2 affords the corresponding five-membered heterocycles, such as $\beta, \gamma$-butenolides (furanones), $\gamma$-imino- $\alpha, \beta$-butenolides, and 1,3-oxazolidin-5-ones which are prepared from $\gamma$-keto acids, maleic acid monoamides, and $N$-acyl- $\alpha$-amino acids, respectively. The reaction of $N$-acylglycines under similar conditions in the presence of aldehydes leads to the formation of 2-alkylidene-1,3-oxazolidin-5-ones. The use of $N$-nitroso- $N$-phenylglycine in place of the $N$-acylglycine leads to 1,2,3-oxadiazoles (sydnones) formation by cyclodehydration. 1,3-Oxazolidine-2-thiones are isolated in excellent to moderate yields when $\beta$-amino alcohols are treated with 2 in the presence of carbon disulfide $\left(\mathrm{CS}_{2}\right)$ as shown in Scheme 2. Treatment of diacylhydrazines with 2 affords the corresponding 1,3,4-oxadiazoles in excellent yields. Self-condensation smoothly occurs when two molar thioamides are treated with 2 in the presence of dimethyl sulfoxide (DMSO), to afford 1,2,4-thiadiazoles in excellent yields. 
Table 2. DMC-mediated cyclodehydration to azetidin-2-ones

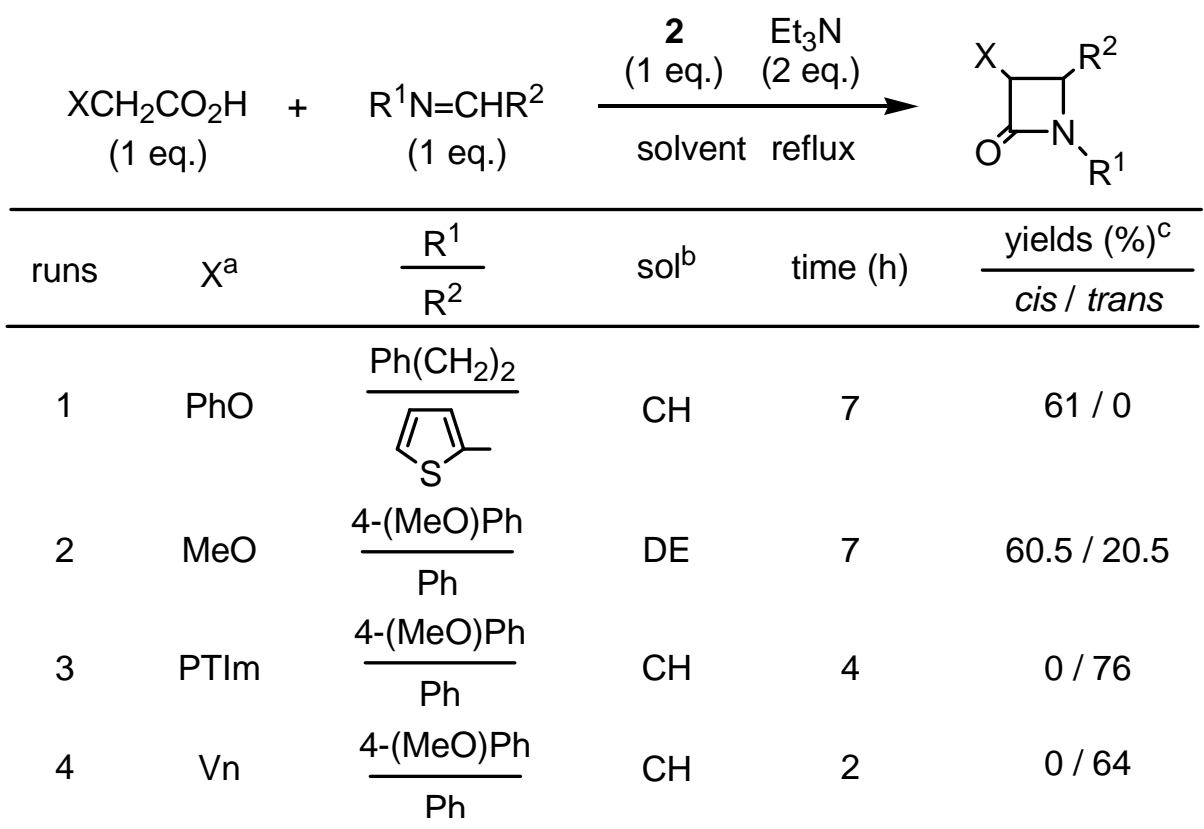

${ }^{\mathrm{a}}$ PTIm=phthalimido; $\mathrm{Vn}=$ vinyl. ${ }^{\mathrm{b}}$ sol=solvent, $\mathrm{CH}=$ chloroform; $\mathrm{DE}=1,2$-dichloroethane. ${ }^{\mathrm{c}}$ Non optimized, isolated yield

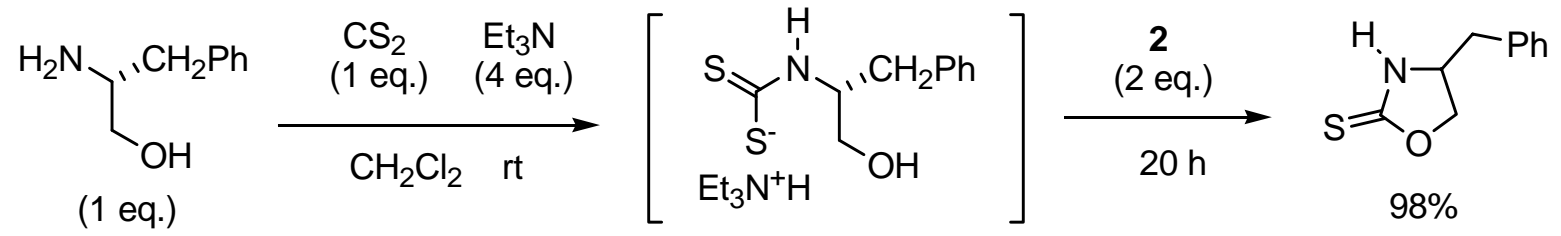

Scheme 2. Preparation of 1,3-oxazolidine-2-thione from $\beta$-amino alcohol

3,1-Benzoxazin-4-ones, six-membered heterocycles, are synthesized in excellent yields by the cyclodehydration of $N$-acylanthranilic acids with 2. (Scheme 3 )

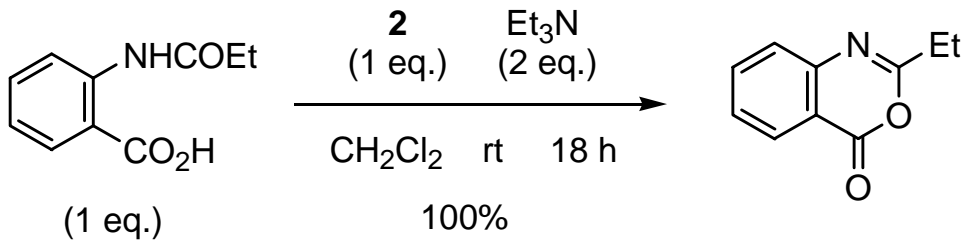

Scheme 3. Cyclodehydration of $N$-acylanthranilic acid to 3,1-benzoxazin-4-one 


\subsection{Chlorination ${ }^{1 \mathrm{c}}$}

DMC (2) can serve as a selective chlorination reagent for primary alcohols.

Treatment of primary alcohols with 2 in dichloromethane at room temperature in the presence of triethylamine affords the corresponding chloroalkanes in excellent yields. (Table 3) This chlorination is also applicable to unsaturated (run 3 in Table 3) or acid-sensitive alcohols containing a carbobenzoxy function (run 5 in Table 3). The use of 1,3-diketones in place of primary alcohols yields vinylogous acid chlorides.

Table 3. DMC-mediated chlorination of primary alcohols

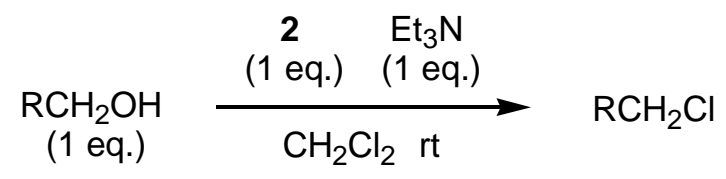

\begin{tabular}{cccc}
\hline runs & $\mathrm{R}$ & time $(\mathrm{h})$ & ${\text { yields }(\%)^{\mathrm{a}}}^{\mathrm{a}}$ \\
\hline 1 & $\mathrm{n}-\mathrm{C}_{7} \mathrm{H}_{15}$ & 12 & 93 \\
2 & $\mathrm{Ph}$ & 17 & 92 \\
3 & $\mathrm{CH}_{2}=\mathrm{CH}\left(\mathrm{CH}_{2}\right)_{7}$ & 21 & 99 \\
4 & $\mathrm{Cl}\left(\mathrm{CH}_{2}\right)_{2} \mathrm{OCH}_{2}$ & 17 & 92 \\
5 & $\mathrm{CbzNHCH}(\mathrm{Bn}) \mathrm{CH}_{2}$ & 16 & 76
\end{tabular}

${ }^{a}$ Non optimized, isolated yield

\subsection{Oxidation ${ }^{1 \mathrm{c}}$}

Secondary ( $\mathrm{sec}$ ) alcohols are oxidized to the corresponding ketones in excellent to good yields by a combination of 2 and DMSO. (Scheme 4) As mentioned above, treatment of a primary benzyl alcohol with 2 in the absence of a coadditive produces the corresponding chloride. Chlorination at the benzylic carbon also predominates in a trial for oxidation of 4-nitrobenzyl alcohol in the presence of DMSO; however, the corresponding aromatic aldehyde is obtained in a moderate yield by the use of hexamethylenetetramine (hexamine) in place of DMSO as an additive after hydrolysis with $50 \%$ aqueous acetic acid.

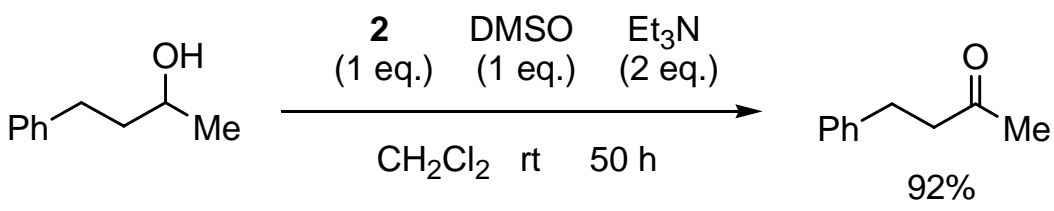

Scheme 4. DMC-Mediated oxidation of sec-alcohol 


\subsection{Reduction $^{1 \mathrm{c}}$}

In the above oxidations of sec-alcohols DMSO should be reduced to dimethyl sulfide. Reduction of dialkyl sulfoxides with 2 to the corresponding sulfides are observed. Interestingly DCC is not effective for the reduction of sulfoxides in spite of the fact that a combination of DCC and DMSO can be used for the oxidation of sec-alcohols, ${ }^{3}$ suggesting that $\mathbf{2}$ is a more powerful reagent than DCC.

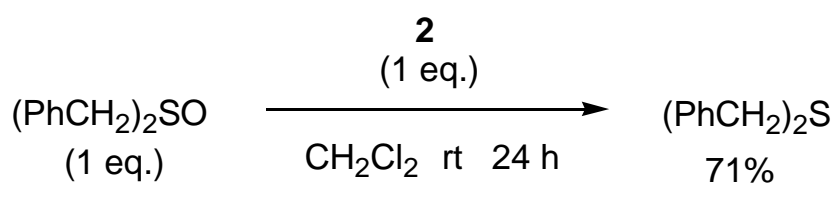

Scheme 5. DMC-mediated reduction of sulfoxide

\subsection{Rearrangement ${ }^{1 \mathrm{c}}$}

Lossen-type rearrangement is observed in the reaction of $\mathbf{2}$ with hydroxamic acids, in which urea, carbamate, and thiocarbamate derivatives are produced in good to moderate yields by trapping intermediate isocyanates with primary amines, alcohols, or thiols, respectively. Smooth Beckmann-like rearrangements are also observed when oximes are used in place of hydroxamic acids. Interestingly a spiro-imine is obtained as a sole product when oximes derived from 4-(4-hydroxy- or 4-methoxyphenyl)-2-butanones are used. (Scheme 6)

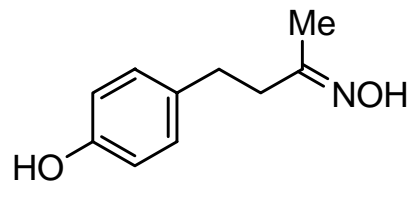

(1 eq.)

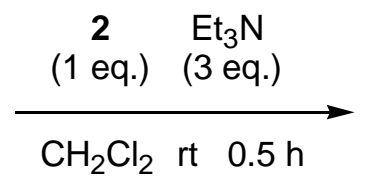

$\mathrm{CH}_{2} \mathrm{Cl}_{2}$ rt $0.5 \mathrm{~h}$

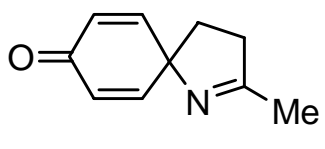

$84 \%$

Scheme 6. DMC-triggered spiro-imine formation

\section{Modified guanidines as chiral auxiliaries}

It is theoretically possible to introduce five chiral centers in a guanidine system. The fact that DMC derivatives react easily with appropriate primary amines to afford cyclic guanidines stimulates us to develop guanidine chemistry, mainly focusing on the role of these guanidines as chiral auxiliaries in asymmetric synthesis. 


\subsection{Preparation ${ }^{4}$}

Modified guanidines are prepared by different methods controlled by the DMC-participating reactions in a key step. The first method involves the reaction of DMC derivatives with amines, by which 1,3-disubstituted 2-iminoimidazolidines 5 and bicyclic guanidines $\mathbf{6}$ are prepared as shown in Scheme 7. ${ }^{4 a}$
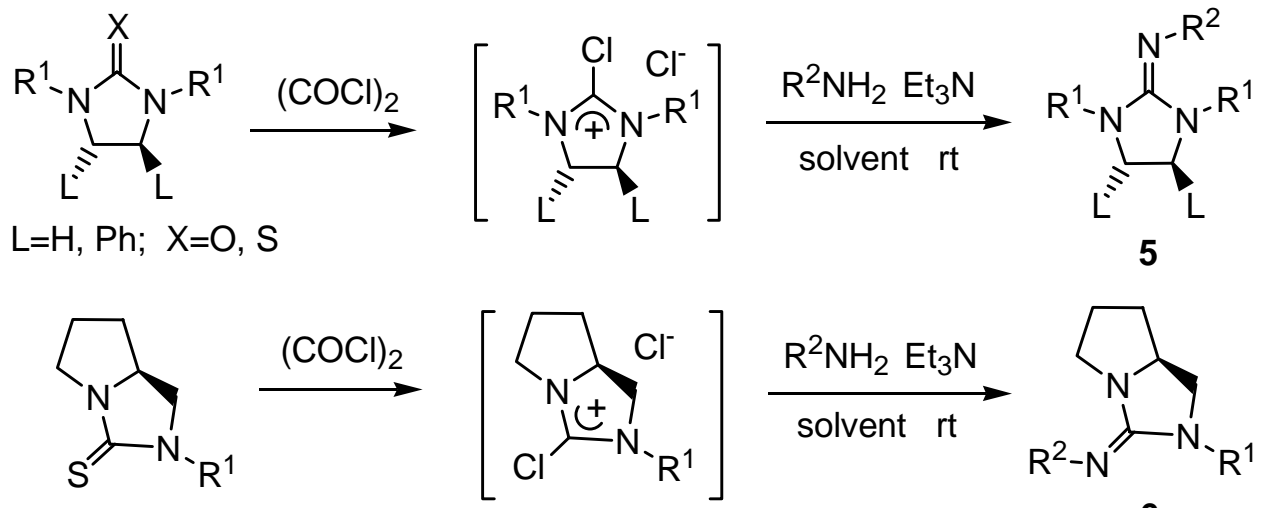

$$
\mathrm{R}^{1}=\mathrm{Me},(S)-1-\text { phenylethyl }
$$

Scheme 7. Preparation of modified guanidines by the amination of DMC derivatives

The second method constitutes DMC-induced cyclization of protected thiourea intermediates 7 derived from the corresponding ethylenediamines. ${ }^{4 b}$ (Scheme 8) Thus, 2-aminoimidazolidines 8 are prepared from trisubstituted thioureas $\left(7: R^{1}, R^{2} \neq H\right)$, whereas 1,3-unsubstituted $\mathbf{9}$ and 1-substitued 2-iminoimidazolidines $\mathbf{1 0}$ are prepared from disubstitued thioureas $\left(7: \mathrm{R}^{2}=\mathrm{H}\right)$ through protected imidazolidine derivatives.

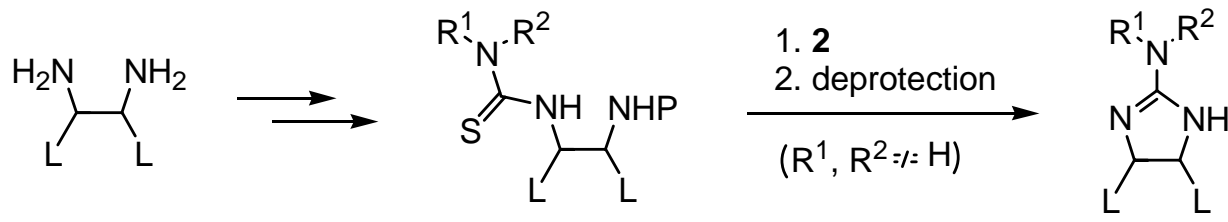

7

$2 \downarrow\left(R^{2}=H\right)$

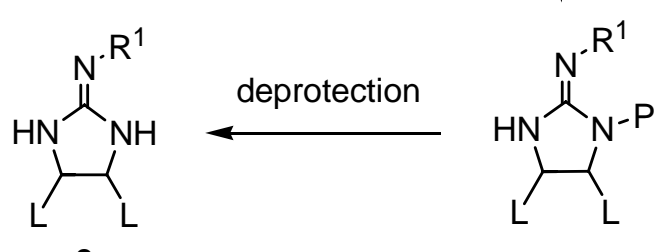

9
1. methylation

2. deprotection

$$
\mathrm{R}^{1}, \mathrm{~L}=\text { alkyl, aryl; } \mathrm{R}^{2}=\text { alkyl, aryl, } \mathrm{H} ; \mathrm{P}=\text { protecting group }
$$

Scheme 8. Preparation of modified guanidines by the DMC-induced cyclization of protected thioureas. 
The third method is shown in Scheme 9, in which an alternative DMC-induced cyclization of guanidines bearing a hydroxyethyl function on the nitrogen atom takes place through the substitution of the hydroxyl group by a chlorine atom. ${ }^{4 c}$
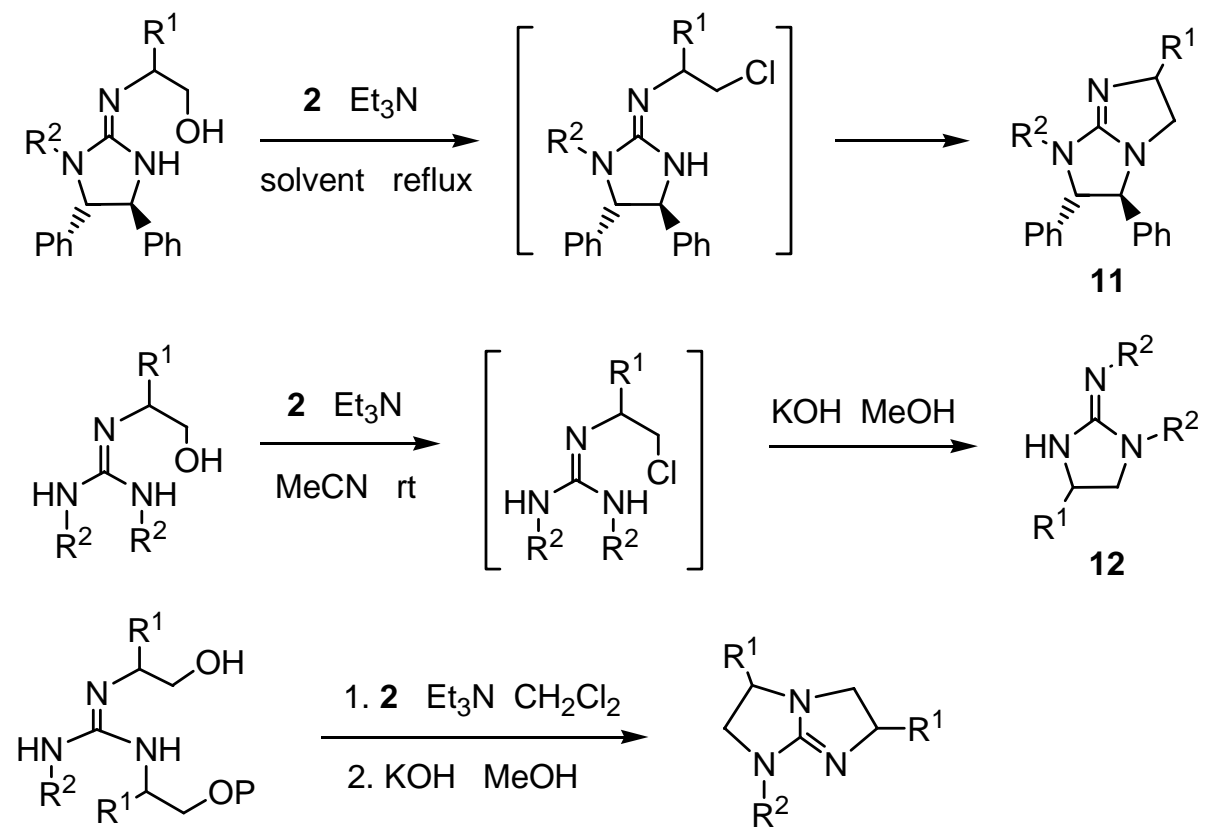

13

$\mathrm{R}^{1}=$ alkyl, aryl; $\mathrm{R}^{2}$ = alkyl, aryl, protecting goup, $\mathrm{H}$ (after deprotection)

Scheme 9. Preparation of modified guanidines by the DMC-induced cyclization of guanidines with a hydroxyethyl function.

Thus, 3,7,8-trisubstituted 1,4,6-triazabicyclooctenes $\mathbf{1 1}$ are prepared from 2-(2-hydroxyethylimino)imidazolidines. 1,4-Disubstituted 2-iminoimidazolidines $\mathbf{1 2}$ are obtained by the reaction of linear-type guanidines with DMC (2) followed by treatment with base. Double DMC-induced cyclization of guanidines containing two 2-hydroxyethyl substituents yields another type of 1,4,6-triazabicyclooctene systems 13.

A combination of the DMC-induced cyclization of thiourea and the intramolecular $\mathrm{S}_{\mathrm{N}} 2$ reaction of monocyclic guanidine formed provides a $C_{2}$-symmetrical bicyclic guanidine $\mathbf{1 4}^{5}$ as shown in Scheme 10. 


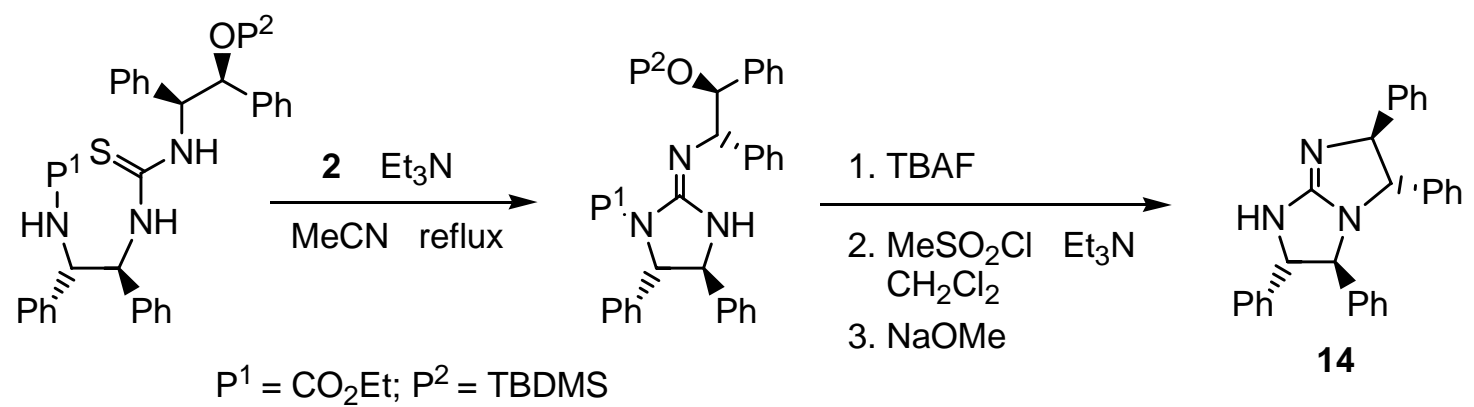

Scheme 10. Preparation of modified guanidines by the DMC-induced cyclization of thiourea followed by $\mathrm{S}_{\mathrm{N}} 2$ reaction

\subsection{Roles as chiral auxiliaries ${ }^{6}$}

Concept of modified guanidines as chiral auxiliaries in asymmetric synthesis is illustrated in Scheme 11, in which reactive, but resonance-stabilized, guanidinium salts 16 could be produced from guanidines 15 by quarternization with an electrophile (A-B).

The salts 16 may react with activated unsaturated substrates $(C=D)$ to give addition products such as in Michael reaction, trimethylsilyl (TMS) cyanation, or nucleophilic epoxidation, in which guanidines should act as catalysts (route A in Scheme 11).<smiles>[R]N=C1N([R])C([Tl])C([Tl])N1[R]</smiles>

15

route $\mathrm{A}$

$\downarrow$ A-B

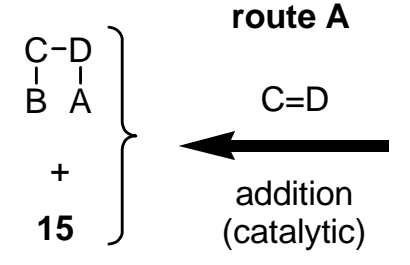

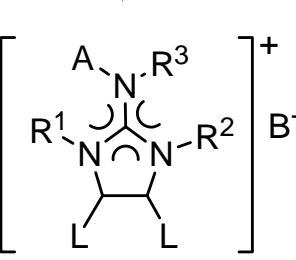

16 route $\mathrm{C}$

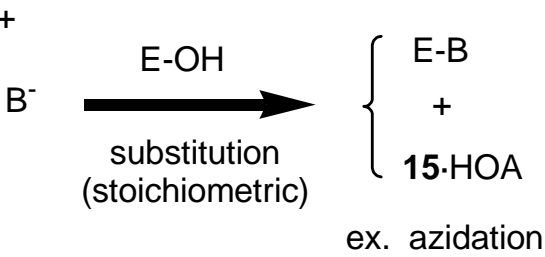

ex. (Michael reaction

TMS cyanation nucleophilic epoxidation

route $B$

$\mathrm{R}^{1}-\mathrm{R}^{3}=$ a possible chiral unit

$$
\begin{aligned}
& \text { EO-A }+\mathbf{1 5} \cdot \mathrm{HB} \\
& \text { ex. }\left\{\begin{array}{l}
\text { silylation } \\
\text { alkylation }
\end{array}\right.
\end{aligned}
$$

Scheme 11. Concept of modified guanidines as chiral auxiliaries 
On the other hand, the guanidinium salts $\mathbf{1 6}$ could be used for kinetic resolution of racemic sec-alcohols $(\mathrm{EOH})$, in which the salt acts as either an electrophile or a nucleophile. Thus, in the former reaction hydrogen-substituted products (EO-A) and protonated guanidines $(\mathbf{1 5} \cdot \mathrm{HB})$ may be produced (route B in Scheme 11), such as in silylation, whereas OH-substituted products (E-B) and alternative protonated guanidines (15. HOA) could be formed in the latter cases (route $\mathrm{C}$ in Scheme 11) such as in azidation. Furthermore, according to route B in Scheme 11, the electrophilic displacement of $\mathbf{1 6}$ with acidic compounds such as carboxylic acids may lead to the resolution of racemic sec-alkyl halides when alkyl halides are used for the guanidinium salt formation as A-B. In these substitution reactions guanidines are needed in stoichiometric amounts.

\subsection{Application to asymmetric synthesis}

Michael reactions between diphenyliminoglycinate $\mathbf{1 7}$ and vinyl compounds $\mathbf{1 8}$ is exemplified as asymmetric synthesis catalyzed by modified guanidines in Table $4 .^{7}$

Table 4. Example of guanidine-catalyzed Michael reaction

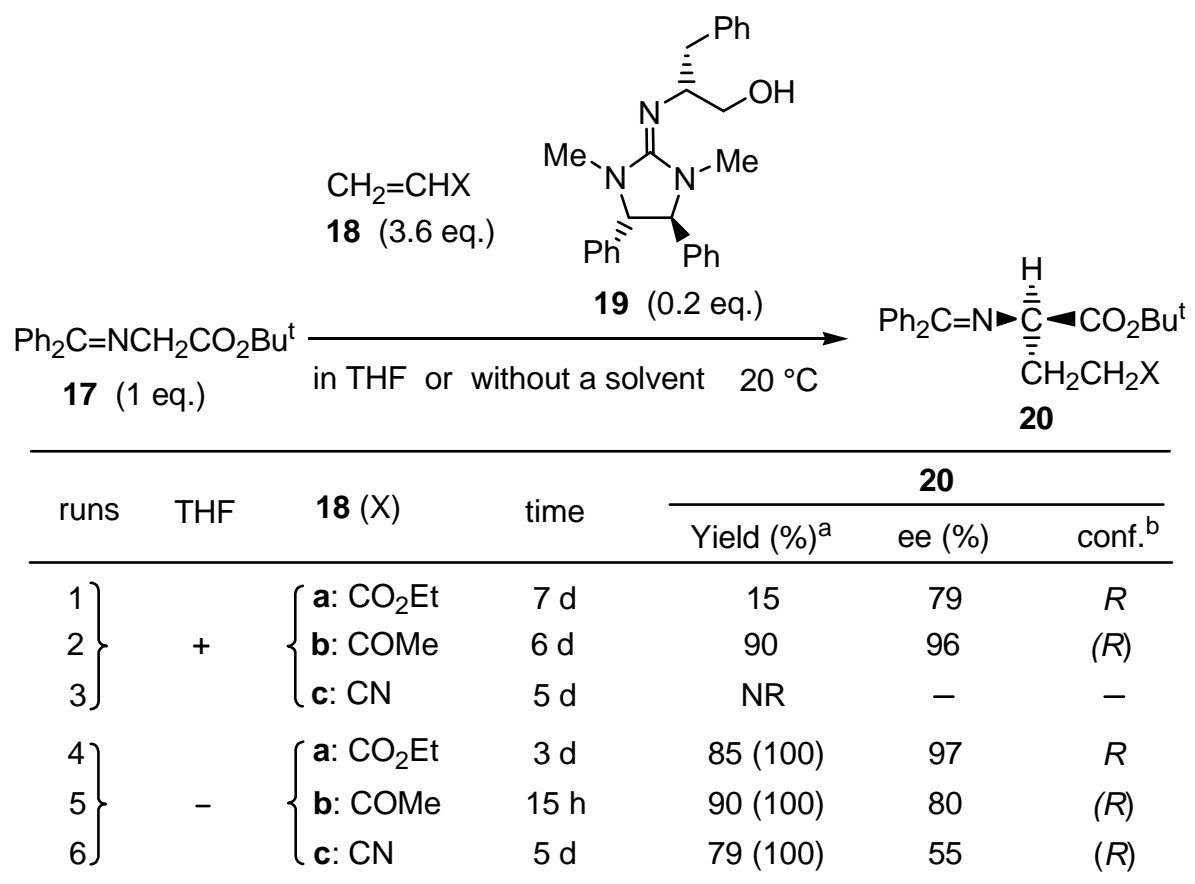

${ }^{a}$ Isolated, non optimized yields. Parentheses show estimation of the product by ${ }^{1}$ HNMR spectra. ${ }^{b}$ Configuration of an excess enantiomer. Parentheses show the expected absolute configuration.

(4S,5S)-1,3-Dimethyl-4,5-diphenyl-2-[(R)-2-hydroxymethyl-2-phenylethylimino]imidazoli dine (19), a monocyclic guanidine [5: $\left.\mathrm{L}=\mathrm{Ph}, \mathrm{R}^{1}=\mathrm{Me}, \mathrm{R}^{2}=(R)-\mathrm{CH}\left(\mathrm{CH}_{2} \mathrm{OH}\right) \mathrm{CH}_{2} \mathrm{Ph}\right]$ prepared by the first method, was found to be the most effective catalyst in this reaction and an excess amount 
of the $(R)$-adduct 20 was obtained. Especially, in the use of ethyl acrylate (18a) as a vinyl compound was quantitatively formed with $97 \%$ ee when the reaction was carried out without solvent (run 4 in Table 4), whereas a less effective reaction (79\% ee in 15\% yield) was observed in tetrahydrofuran (THF) solution (run 1 in Table 4). Interestingly, replacement of the acrylate 18a to methyl vinyl ketone (18b) led to opposite results (runs 2 and 5 in Table 4) and, thus, a higher asymmetric induction ( $96 \%$ ee) was observed in THF solution even with longer reaction time (90\% yield after 6 days). In general, remarkable rate acceleration was observed in solvent-less reactions (runs 4-6 in Table 4).

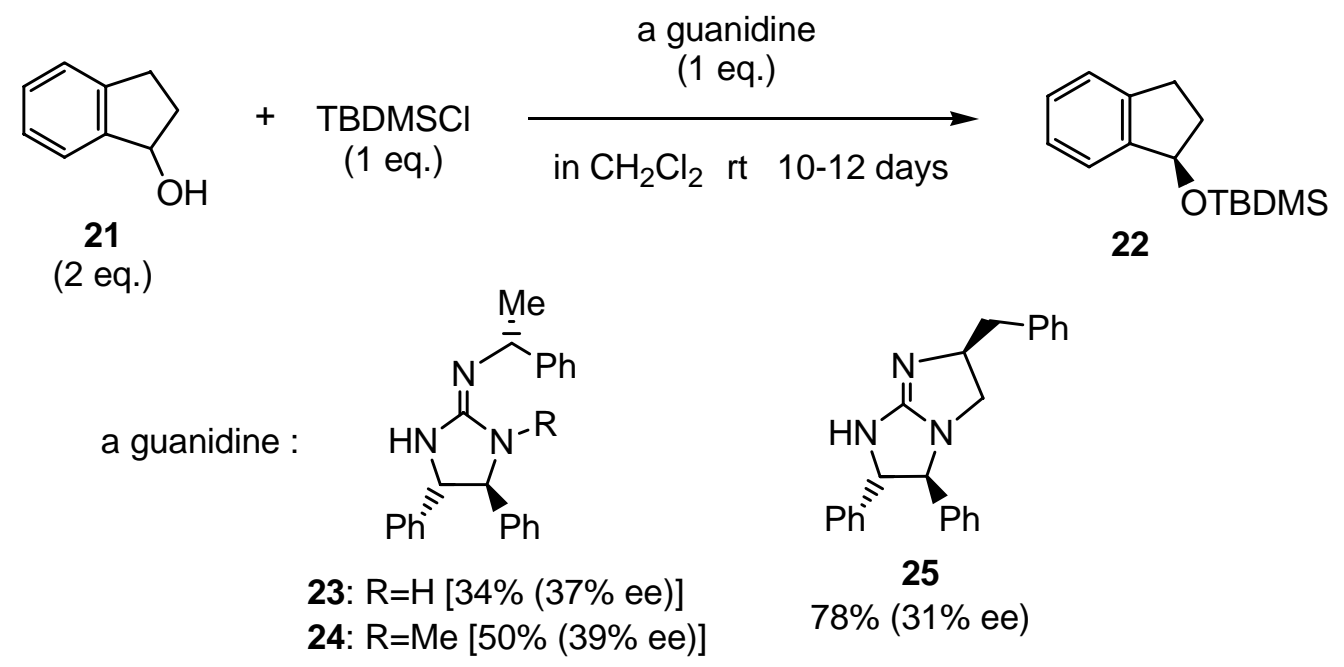

Scheme 12. Guanidine-induced kinetic resolution of sec-alcohol

Kinetic resolution of sec-alcohols by silylation ${ }^{8}$ was exemplified as a stoichiometric reaction. $( \pm$ )-Indanol (21) was treated with tert-butyldimethylsilyl chloride (TBDMSCl) in the presence of a variety of modified guanidines. (Scheme 12) 1,3-Unsubstituted guanidine 23 [9: $\mathrm{L}=\mathrm{Ph}, \mathrm{R}^{1}=$ $(R)-\mathrm{CH}(\mathrm{Me}) \mathrm{Ph}]$, its 1-methyl derivative $24\left[10: \mathrm{L}=\mathrm{Ph}, \mathrm{R}^{1}=(R)-\mathrm{CH}(\mathrm{Me}) \mathrm{Ph}\right]$, or $(3 S, 7 S, 8 S)$-3-benzyl-7,8-diphenyl-1,4,6-triazabicyclo[3.3.0]oct-4-ene $\quad(25) \quad\left[11: \quad \mathrm{R}^{1}=\right.$ $(S)-\mathrm{CH}(\mathrm{Me}) \mathrm{Ph}, \mathrm{R}^{2}=\mathrm{H}$ ] were found to be effective auxiliaries. Higher ee $(\approx 70 \%)$ was observed when a more bulky triisopropylsilyl chloride was used as a silylating agent in place of TBDMSCl.

\section{Guanidinium ylides for aziridine formation}

Guanidinium ylides have never been known in the literature to our knowledge; however, these would be expected to act as stabilized equivalents of azomethine ylides due to the resonance stabilization of the cationic nitrogen function. In the course of our studies on guanidine chemistry 
we found that treatment of guanidinium salts 26, containing a glycine unit, with aryl aldehydes in the presence of a base directly afforded 3-arylaziridine-2-carboxylates $\mathbf{2 8}$ in high yields with excellent to moderate trans diastereoselectivity together with a recyclable DMI derivative 29. (Scheme 13) Furthermore, the introduction of chiral centers into the guanidinium template (26: $\mathrm{L}=$ $\mathrm{Ph}$ ) resulted in effective asymmetric induction on the aziridine formation. In this reaction guanidinium ylides 27 derived form guanidinium salts $\mathbf{2 6}$ by base treatment should act as electrophiles.

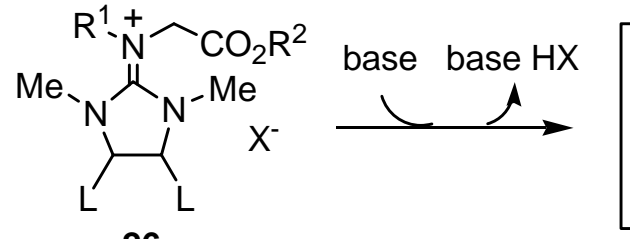

26

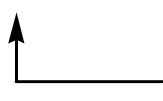

amination<smiles>CN1C(Cl)=[N+](C)C(I)C1I</smiles>

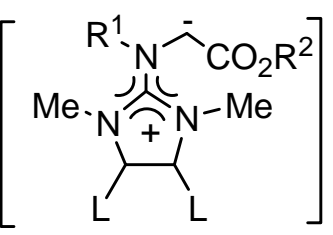

27

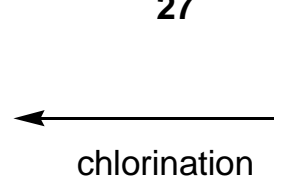

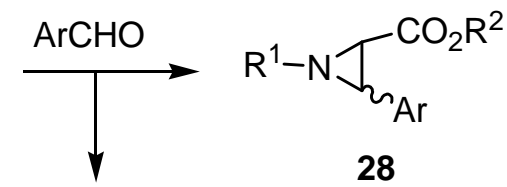

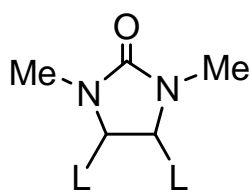

29

Scheme 13. Schematic aziridine formation by the reaction of guanidinium ylide with an aryl aldehyde

\subsection{Reaction using achiral guanidinium ylides ${ }^{9}$}

Reaction of guanidinium bromide 30 with aryl aldehyde in dimethylformamide (DMF) in the presence of sodium hydride $(\mathrm{NaH})$ followed by treatment with silica gel $\left(\mathrm{SiO}_{2}\right)$ affords 3-arylaziridine-2-carboxylate 31 in reasonable yield together with DMI (1). The aziridine product $\mathbf{3 1}$ is similarly yielded when a mixture of $\mathbf{3 0}$ and aryl aldehyde is stirred with a slight excess of tetramethylguanidine (TMG).

This synthetic method is widely applicable to a variety of aryl aldehydes, including heterocycles, as shown in Table 5, in which trans aziridines trans-31 are preferentially formed. In particular, not only trans selectivity ( $c$ a 90\% de) but also satisfactory conversion (68-95\%) are observed when electron-rich benzaldehydes (runs 3-6 in Table 5) and indolyl aldehydes (runs 12 and 13 in Table 5) are used. Cinnamaldehyde could also be converted to 1,2-addition-type aziridine products $\mathbf{3 1 g}$ in total 70\% yield. (run 11 in Table 5) Thus, this reaction sequence could be regarded as an effective cycle reaction as shown in Scheme 12, because of no waste of any key components during reactions. 
Table 5. Reaction of achiral guanidinium salt with aryl aldehydes

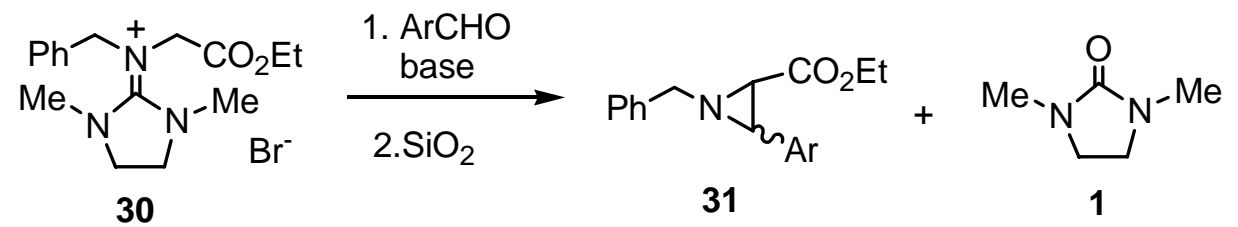

\begin{tabular}{|c|c|c|c|c|c|c|c|}
\hline \multirow{2}{*}{ runs } & \multirow{2}{*}{$\operatorname{Ar}$} & \multirow{2}{*}{ base $^{a}$} & \multirow{2}{*}{$\begin{array}{l}\text { time } \\
\text { (h) }\end{array}$} & \multicolumn{3}{|c|}{ yield $(\%)^{b}$ of 31} & \multirow{2}{*}{$\begin{array}{c}1 \\
(\%)\end{array}$} \\
\hline & & & & cis & trans & cis / trans & \\
\hline 1) & \multirow[b]{2}{*}{ a } & $\int A$ & 24 & $\int 23$ & 61 & $27 / 73$ & 78 \\
\hline 2$\}$ & & B & 24 & $\{28$ & 41 & $41 / 59$ & 77 \\
\hline 3) & \multirow{2}{*}{$b$} & $\int A$ & 24 & $\{8$ & 69 & $10 / 90$ & 71 \\
\hline $4 \int$ & & $B$ & 24 & 4 & 64 & $6 / 94$ & 83 \\
\hline 5 & c & $A$ & 9 & 7 & 78 & $8 / 92$ & $-c$ \\
\hline 6 & $d$ & $A$ & 8 & 2 & $93^{d}$ & $2 / 98$ & $-c$ \\
\hline 7) & \multirow[b]{2}{*}{ e } & $\int A$ & 24 & $\{17$ & 48 & $26 / 74$ & 93 \\
\hline 8$\}$ & & B & 24 & $l_{16}$ & 45 & $26 / 74$ & 94 \\
\hline 9$]$ & \multirow{2}{*}{$f$} & $A$ & 24 & 2 & 45 & $4 / 96$ & $-c$ \\
\hline 10 & & B & 2 & 3 & 43 & $7 / 93$ & 72 \\
\hline 11 & g & $A$ & 24 & 17 & $53^{d}$ & $24 / 76$ & $-c$ \\
\hline 12 & $\mathrm{~h}$ & $A$ & 24 & 4 & $72^{d}$ & $5 / 95$ & 88 \\
\hline 13 & i & $A$ & 24 & 4 & $75^{d}$ & $5 / 95$ & 88 \\
\hline 14 & j & $A$ & 7 & 8 & 58 & $12 / 88$ & 72 \\
\hline
\end{tabular}

${ }^{\mathrm{a}} \mathrm{A}$ : NaH. The reaction was carried out in DMF at $-20{ }^{\circ} \mathrm{C}$. B: TMG. The reaction was carried out without solvent at $\mathrm{rt} .{ }^{\mathrm{b}}$ Isolated, non-optimixed yield. ${ }^{\mathrm{c}}$ Isolation was not attempted. ${ }^{\mathrm{d}}$ A mixture of two invertomers at the nitrogen atom.

\subsection{Appliaction to asymmetric synthesis ${ }^{9}$}

This aziridine synthesis is applicable to asymmetric synthesis. (Table 6) Smooth reaction is observed when the chiral guanidinium bromide 32 is treated with aryl aldehydes under the same conditions as achiral salts, in which TMG (run 3 in Table 6) is more effective in both chemical yield and stereoselectivity than $\mathrm{NaH}$ in DMF (run 4 in Table 6). Introduction of chiral units into the imidazolidine ring could cause rate acceleration of the reaction and effective product formation with satisfactory enantioselectivity, in which DMC derivative $\mathbf{3 4}$ is, as expected, recovered as a reusable source for the chiral guanidinium template 32.

Chemical correlation of 3-phenylaziridine-2-carboxylates (cis-33a: 79\% ee; trans-33a: 77\% 
ee) obtained in run 1 in Table 6 with commercially available (S)-phenylalaninate 36, as shown in Scheme 14 , indicates that the $(S, S)$-guanidinium ylide $(S, S)$-32 mainly produces $(2 R, 3 R)$-cis and $(2 R, 3 S)$-trans aziridines. These facts strongly suggest that the $\mathrm{C} 2$ stereogenic centers in the cis and trans aziridine skeletons have to be controlled in the same fashion. In other words, it is $\mathrm{C}$ - $\mathrm{C}$ bond formation, but not N-C bond formation, that should play a crucial role for asymmetric induction in this reaction between guanidinium ylide and aryl aldehyde.

Table 6. Asymmetric aziridine synthesis using chiral guanidinium bromide

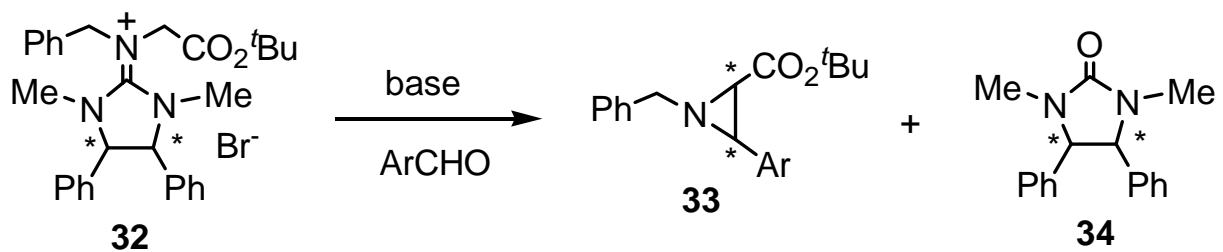

\begin{tabular}{|c|c|c|c|c|c|c|c|c|}
\hline \multirow{2}{*}{ runs } & \multirow{2}{*}{32} & \multirow{2}{*}{$\mathrm{Ar}$} & \multirow{2}{*}{ base } & \multirow{2}{*}{$\begin{array}{l}\text { time } \\
\text { (h) }\end{array}$} & \multicolumn{3}{|c|}{ yield $^{b}(\%)$ of $33^{c}$} & \multirow{2}{*}{$\begin{array}{c}34 \\
(\%)\end{array}$} \\
\hline & & & & & cis (ee\%) & trans (ee\%) & cis / trans & \\
\hline 1 & $(S, S)$ & $\mathbf{a}$ & B & 3 & $60(79)$ & 31 (77) & $66 / 34$ & 88 \\
\hline 2 & $(R, R)$ & $\mathbf{a}$ & B & 5 & $61(75)$ & $32(73)$ & $66 / 34$ & 96 \\
\hline 3 & $(S, S)$ & b & B & 4 & $6(-)$ & 82 (97) & $7 / 93$ & 91 \\
\hline 4 & $(R, R)$ & b & A & 39 & $7(-)$ & $75(72)$ & $9 / 91$ & 86 \\
\hline 5 & $(S, S)$ & e & B & 4 & $51(-)$ & 35 (59) & $59 / 41$ & $-d$ \\
\hline 6 & $(S, S)$ & $\mathbf{h}$ & B & 7 & $6(-)$ & $70(95)$ & $8 / 92$ & 87 \\
\hline 7 & $(S, S)$ & $\mathbf{i}$ & $B$ & 5.5 & $9(-)$ & $87(76)$ & 9 / 91 & 90 \\
\hline
\end{tabular}

a The reaction was carried out under the same conditions shown in Table 5. ${ }^{b}$ Isolated non-optimized yield. ${ }^{\mathrm{c}}$ Ee was determined by chiral HPLC. ${ }^{\mathrm{d}}$ Isolation was not attempted.

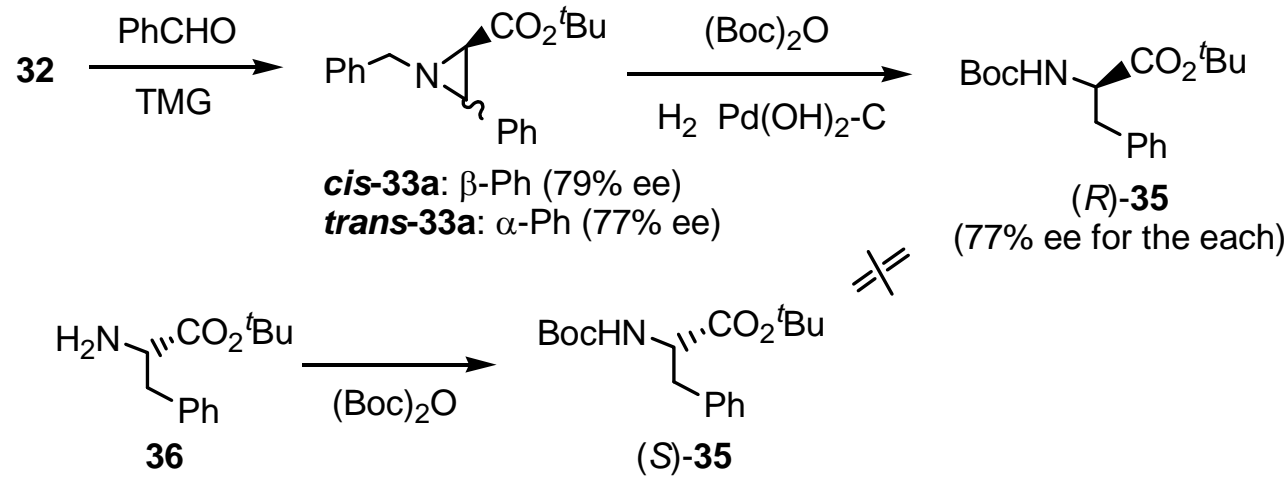

Scheme 14. Determination of the absolute stereochemistry of aziridine products 


\subsection{Mechanistic approaches ${ }^{10}$}

Next, we approached a reasonable mechanism for this guanidinium ylide-participated asymmetric aziridine synthesis. Overall reaction is composed of two steps as shown in Scheme 15. Step 1 is a $\mathrm{C}-\mathrm{C}$ bond formation between 32 and an aryl aldehyde under basic conditions in which an initially-formed zwitter ionic species $\mathbf{3 7}$ is in equilibria with a non-ionic spiro compound $\mathbf{3 8}$. The second step is the fragmentation of the intermediate adduct, triggered with $\mathrm{SiO}_{2}$, to afford aziridine product $\mathbf{3 3}$ and urea $\mathbf{3 4 .}$

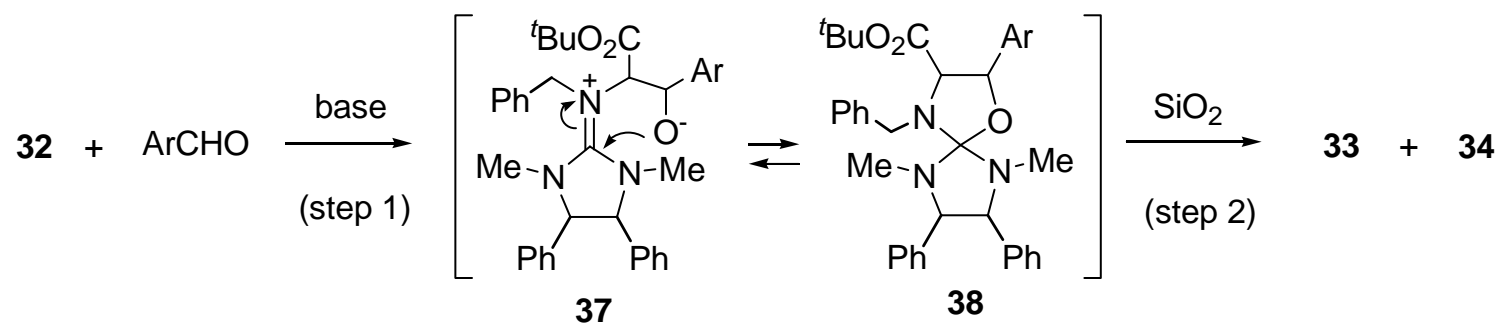

Scheme 15. Two steps mechanism for asymmetric aziridine synthesis

We found, in the reaction of 32 and piperonal (3,4-methylenedioxybenzaldehyde), that treatment with acetic anhydride $\left(\mathrm{Ac}_{2} \mathrm{O}\right)$, as well as $\mathrm{SiO}_{2}$, immediately converts the intermediate adduct into the trans aziridine trans-33 in $86 \%$ yield with $87 \%$ ee (cis-33 in $7 \%$ yield) together with urea 34 (80\% yield). These facts prompt us to further examine the effect on the aziridine synthesis of various substituents in the para position of benzaldehyde under modified conditions using $\mathrm{Ac}_{2} \mathrm{O}$. After stirring a mixture of 32 and a benzaldehyde derivative in THF in the presence of TMG at $25^{\circ} \mathrm{C}$ for $5 \mathrm{~h}$ (step 1), the intermediate adduct is treated with $\mathrm{Ac}_{2} \mathrm{O}$ at room temperature until disappearance of the polar intermediate on thin layer chromatography (TLC) (step 2). The results are summarized in Table 7.

No reaction occurs when $p$-dimethylaminobenzaldehyde is used (run 1 in Table 7). Although in the cases of alkoxy-substituted benzaldehydes starting aldehydes remain in step 1, the intermediate adducts are immediately transformed in step 2 (runs 2 and 3 in Table 7). In these reasctions the trans derivatives trans-33 are produced as major aziridine isomers with excellent stereoselectivity in both ee and de. $p$-Tolualdehyde and benzaldehyde itself smoothly afford aziridines 33 with high asymmetric inductions (runs 4 and 5 in Table 7). Interestingly, in those cases, the major isomers are cis derivatives. The nearly same results are obtained in runs 6 and 7 when $p$-chloro- and p-methoxycarbonylbenzaldehydes are used. The main difference is the reaction rate of step 2; slower reactions in runs 6 and 7 (about $17 \mathrm{~h}$ ) compared to runs 4 and $5(<3$ h). On the other hand in the cases of p-cyano- and p-nitrobenzaldehydes, the asymmetric induction of aziridines is greatly lowered and, in addition, the predominant formation of trans aziridine 
trans-33 is again observed (runs 8 and 9 in Table 7).

Table 7. Reactions of $(S, S)$-guanidinium salt and various $p$-substituted benzaldehydes

\begin{tabular}{|c|c|c|c|c|c|c|c|c|}
\hline \multirow{2}{*}{$\begin{array}{r}32+ \\
\text { runs }\end{array}$} & \multicolumn{3}{|c|}{$\begin{array}{l}\text { TMG (1.1 eq.) } \\
\underset{2}{\stackrel{\text { in THF }}{\longrightarrow}} \\
\stackrel{{ }^{\circ} \mathrm{C}, 5 \mathrm{~h}}{\text { (step } 1)}\end{array}$} & adduct] & $\begin{array}{l}\mathrm{Ac}_{2} \mathrm{O}(2.2 \text { eq.) } \\
\underset{\mathrm{CHCl}_{3} \mathrm{rt}}{(\text { step 2) }}\end{array}$ & 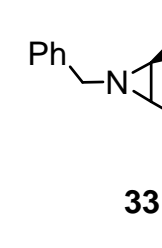 & $\mathrm{CO}_{2}{ }^{t} \mathrm{BL}$ & +34 \\
\hline & $\begin{array}{c}X \text { in } \\
\mathrm{ArCHO}\end{array}$ & $\begin{array}{l}\text { time }(\mathrm{h}) \\
\text { in step } 2\end{array}$ & $\begin{array}{c}\text { total } 33 \\
(\%)^{\mathrm{a}}\end{array}$ & $\begin{array}{c}\text { trans-33 } \\
(\%)^{\mathrm{a}}(\mathrm{ee})^{\mathrm{b}}\end{array}$ & $\begin{array}{c}\text { cis-33 } \\
(\%)^{\mathrm{a}}(\mathrm{ee})^{\mathrm{b}}\end{array}$ & $\begin{array}{c}\text { ratio of } \\
\text { trans-33 } \\
(\%)^{\mathrm{a}}\end{array}$ & $\begin{array}{c}33 \\
(\%)^{\mathrm{a}}\end{array}$ & $\begin{array}{c}\mathrm{ArCHO} \\
(\%)^{\mathrm{a}}\end{array}$ \\
\hline 1 & $\mathrm{NMe}_{2}$ & 0.5 & $-d$ & - & - & - & - & 95 \\
\hline 2 & $\mathrm{O}^{n} \mathrm{Bu}$ & 0.5 & 67 & $64(92)$ & $3(-)$ & 96 & 81 & 29 \\
\hline 3 & OMe & 0.5 & 81 & 77 (91) & $4(-)$ & 95 & 65 & 12 \\
\hline 4 & $\mathrm{Me}$ & 1.5 & 76 & $31(93)$ & $45(90)$ & 41 & 90 & none \\
\hline 5 & $\mathrm{H}$ & 3 & 80 & $22(88)$ & $58(86)$ & 28 & 100 & none \\
\hline 6 & $\mathrm{Cl}$ & 17 & 92 & $33(84)$ & $59(86)$ & 36 & $-^{f}$ & none \\
\hline 7 & $\mathrm{CO}_{2} \mathrm{Me}$ & 17.5 & 80 & $28(72)$ & $52(79)$ & 35 & 69 & none \\
\hline 8 & $\mathrm{CN}$ & 17.5 & 53 & 35 (32) & $18(16)$ & 66 & 50 & none \\
\hline 9 & $\mathrm{NO}_{2}$ & 49 & 70 & $41(11)$ & $29(10)$ & 59 & 67 & none \\
\hline
\end{tabular}

${ }^{a}$ Isolated yield. ${ }^{b}$ Determined by chiral HPLC. ${ }^{c}$ (trans-6/total 6) $\times 100 .{ }^{d}$ No aziridine is formed even with an excess of the aldehyde. ${ }^{\mathrm{e}}$ Not measured. ${ }^{\mathrm{f}}$ not purified.

Therefore, these aziridine formations could be categorized into four groups based on the nature of aldehyde substrates; (i) group A, which includes benzaldehydes with a strong EDG (X= $\mathrm{O}^{\mathrm{n}} \mathrm{Bu}$ and $\mathrm{OMe}$ ), is characterized by a very slow step 1, a very rapid step 2, and excellent diastereoselectivity and enantioselectivity of trans aziridine; (ii) group B is represented by a slow step 1 and a rapid step 2 with moderate cis diastereoselectivity and excellent to good enantioselectivity in both aziridines, and contains benzaldehydes which possess a weak EDG (X) $\mathrm{Me}$ ) or no substituents $(X=H)$; (iii) group $C$ shows a similar profile as in group $B$ but with a rapid step 1 and a slow step 2, and comprises benzaldehydes with a weak EWG $\left(\mathrm{X}=\mathrm{Cl}\right.$ and $\left.\mathrm{CO}_{2} \mathrm{Me}\right)$, and (iv) group $\mathrm{D}$, which includes benzaldehydes with a strong $\mathrm{EWG}\left(\mathrm{X}=\mathrm{CN}\right.$ and $\mathrm{NO}_{2}$ ), is characterized by a moderate trans diastereoselectivity and low enantioselectivity with a very rapid step 1 and a very slow step 2 . 


\subsection{Proposed mechanisms ${ }^{10}$}

Nearly exclusive formation of trans-(2R,3S)-33 with excellent enantioselectivity in group A could be explained by mechanism shown in Scheme 16. An aldehyde approaches to the less hindered $\operatorname{Re}$-face $[\operatorname{Re}(\mathrm{G})]$ of ylide enolate because the $\mathrm{Si}$-face is blocked by $N$-Me group located in the stable $Z$-configuration of the $\mathrm{C}=\mathrm{N}^{+}$double bond. Thus, $R$ stereochemistry of stereogenic center in the $\mathrm{C}-\mathrm{C}$ bond formation should be induced. The step 1 is very slow due to the lowered electrophilicity of the carbonyl function of aldehyde substrates due to the resonance stabilization of $p$-substituted alkoxy group. Thus, the less hindered $\operatorname{Re}$-face $[\operatorname{Re}(\mathrm{A})]$ of aldehyde greatly participates to the bond formation, resulting in the production of a stable $(4 R, 5 S)$-oxazolidine 39 with a trans configuration between the ester and the aryl groups. Then, very rapid concerted fragmentation like $\mathrm{S}_{\mathrm{N}} \mathrm{i}$ type reaction can occur with retention at the $\mathrm{C}-5$ benzylic carbon to give trans-( $2 R, 3 S)$-aziridine trans-(2R,3S)-33. It is reasonable to expect that $\mathrm{Ac}_{2} \mathrm{O}$ could coordinate the oxygen atom of the oxazolidine moiety in $\mathbf{3 9}$ and the partially cationic character of the oxygen atom in the coordinated species initiates the fragmentation of the intermediate adduct to trans-33 and a 2-acetoxyamidinium salt, convertible to urea 34, by simultaneous reactions of the O-C(Ar) bond cleavage and the $\mathrm{N}$ (oxazolidine)-C(Ar) bond formation. In this reaction sequence partially-generated benzyl cationic species could be stabilized by the electron-relayed participation of $p$-substituted alkoxy group. Efficient synthesis of trans aziridines with excellent stereoselectivity is due to a combination of activation with $\mathrm{Ac}_{2} \mathrm{O}$ and the electronic stabilization by the EDG, the former activation of which acts as a rate-determining step in the fragmentation.

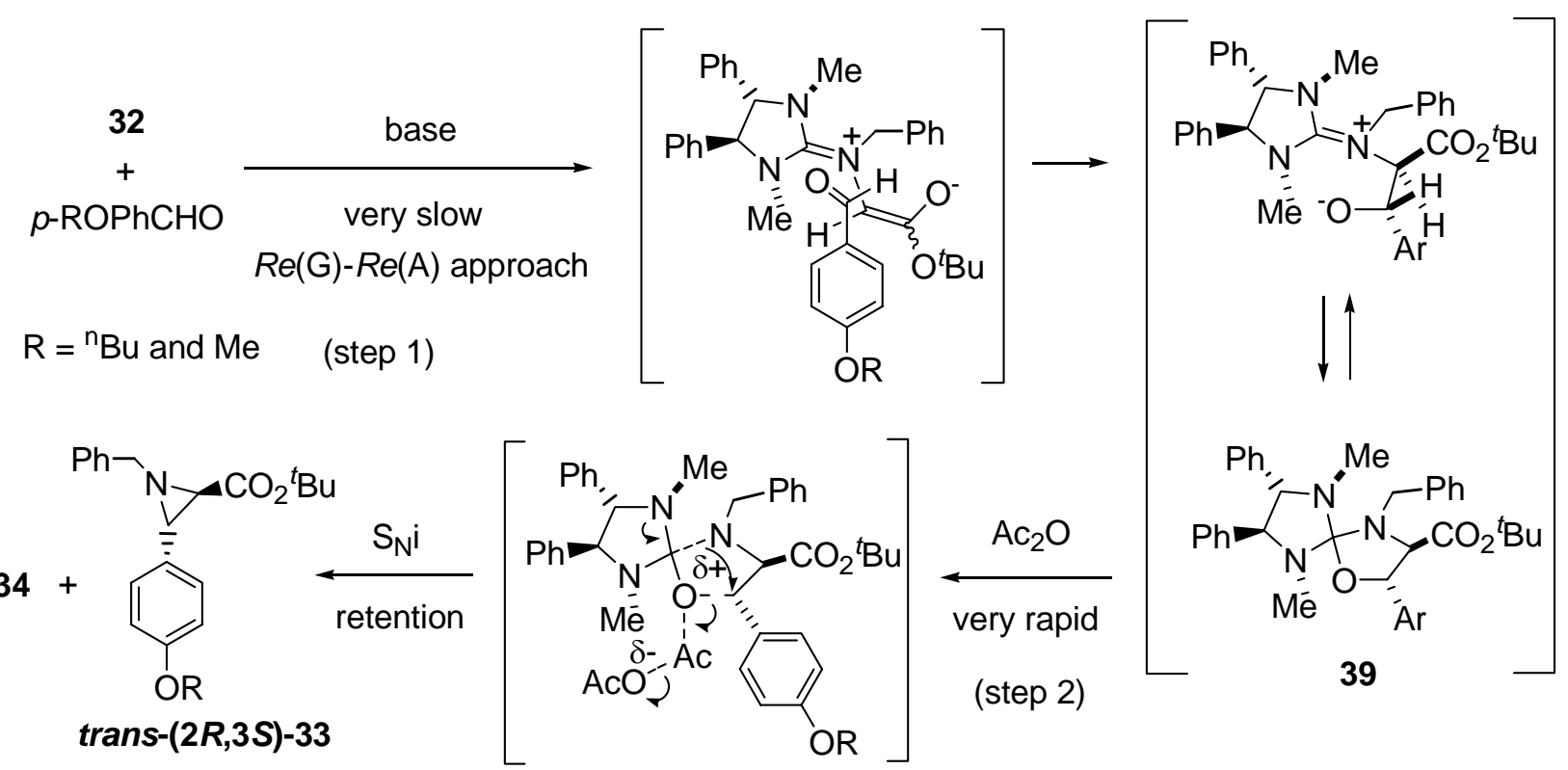

Scheme 16. Proposed mechanism for group A 
Group C carrying a weak EWG shows moderate diastereoselectivity, but with good enantioselectivity, similar to group B either with a weak EDG or without substituents. As mentioned above, major difference between group $\mathrm{B}$ and $\mathrm{C}$ is reaction time needed for the completion of the fragmentation (step 2). Since steric environment is all identical around the reaction sites, this fact suggests a different rate-determining step during the fragmentation. In group $\mathrm{C}$, rapid reaction in step 1 allows an alternative $\operatorname{Re}(\mathrm{G})-\operatorname{Si}(\mathrm{A})$ approach in addition to a major $\operatorname{Re}(\mathrm{G})-\operatorname{Re}(\mathrm{A})$ approach, resulting in lowered face selectivity. The oxygen atom of the oxazolidine unit in a diastereomeric mixture of spiro intermediates $\mathbf{4 0}$ is similarly activated with $\mathrm{Ac}_{2} \mathrm{O}$; however, the benzyl cationic species supposed in Scheme 16 could not be formed because of destabilization with EWG. Therefore, in this case the cleavage of the $\mathrm{C}$ (spiro)-N(oxazolidine) bond involving the nitrogen atom of the imidazolidine unit affords an opened amidinium intermediate 41, in which intramolecular $\mathrm{S}_{\mathrm{N}} 2$ type reaction occurs, thus, resulting in the predominant production of cis- $(2 R, 3 R)$-aziridine cis-(2R,3R)-33 from a major trans-(4R,5S)-oxazolidine $\mathbf{4 0}$ with inversion of the configuration at C-5. (Scheme 17) The slightly lowered ee of aziridines could be explained by competitively enantiofacial accesses, such as the $\operatorname{Si}(\mathrm{G})-\operatorname{Re}(\mathrm{A})$ approach, in rapid reaction of step 1.

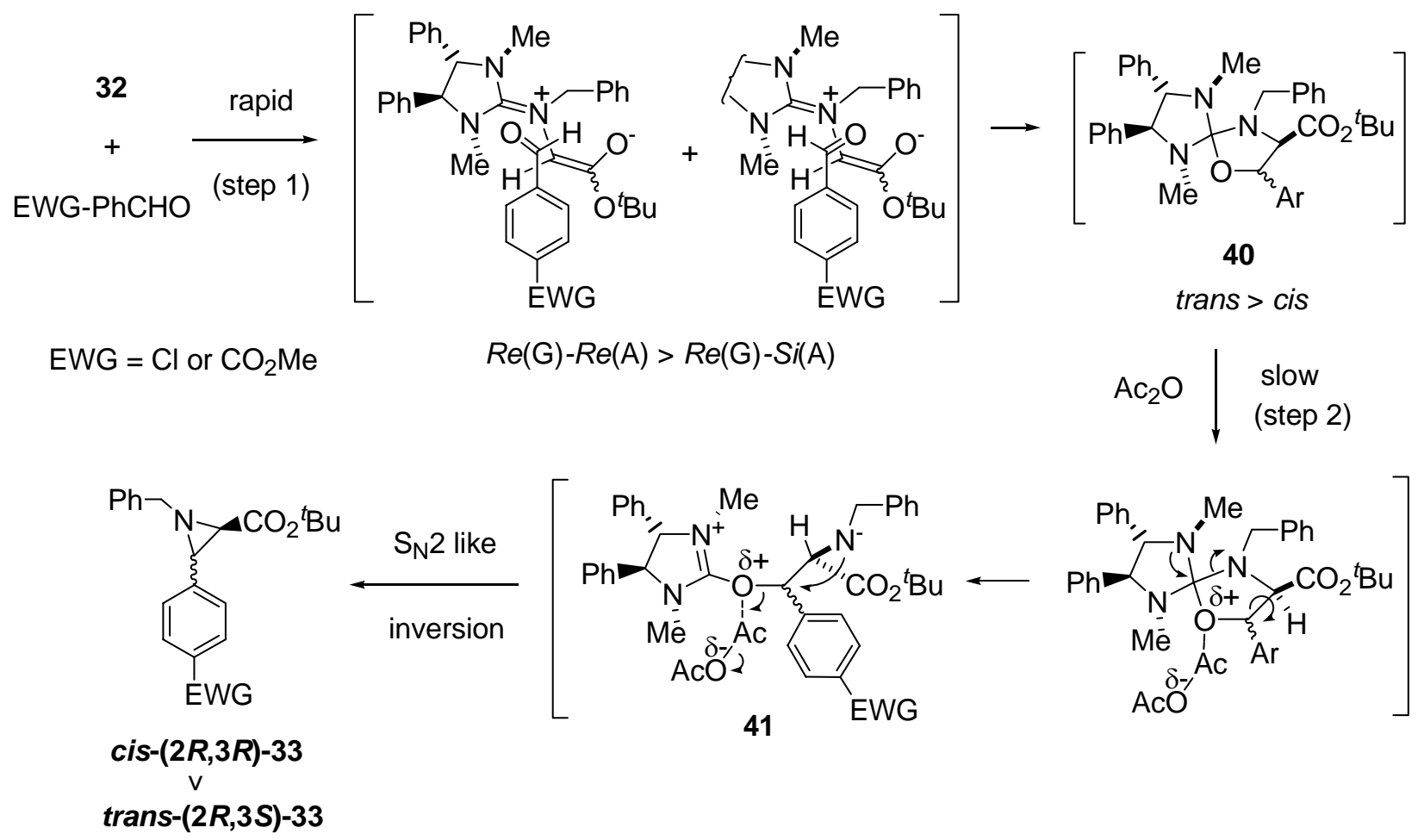

Scheme 17. Proposed mechanism for group C 
Excellent to good ee observed in group B are explained by the high enantiofacial selectivity of the $\operatorname{Re}(\mathrm{G})-\operatorname{Re}(\mathrm{A})$ approach in slow reaction of step 1 as observed in group A (see, Scheme 16); however, the lowered diastereoselectivity could not be covered by application of mechanism proposed for group A. The reduction of the electron-donating effect and the longer reaction time of step 2 in group $B$ than that in group A suggest the participation of both $\mathrm{S}_{\mathrm{N}} \mathrm{i}$ and $\mathrm{S}_{\mathrm{N}} 2$ type reactions (see, Schemes 16 and 17), causing lower diastereoselectivity in group B.

In group D with a strong EWG not only the great reduction of stereoselectivity in aziridine production but also the unexpected formation of the trans-aziridine can be explained by a very rapid step 1, in which four competitive approaches produce all possible isomers and a very slow step 2, in which amidinium intermediates (see, 41 in Scheme 17) are in equilibrium to spiro intermediates (see, $\mathbf{4 0}$ in Scheme 17), different from the three reactions mentioned above.

Hammett approaches are examined for more quantitative analysis. A first Hammett analysis, based on diastereomeric ratios in aziridine products, is consistent with the proposed rate-determining steps in these two $\mathrm{S}_{\mathrm{N}} \mathrm{i}$ and $\mathrm{S}_{\mathrm{N}} 2$ type mechanisms. A second Hammett analysis, based on the enantiomeric ratio of the aziridine products, reveals difference in susceptibilities to electronic substituent effect between the nucleophilic addition (step 1) and the intramolecular nucleophilic substitution (step 2).

\section{Conclusions}

Thus, our roads to guanidine chemistry originated from DMI (1) have been discussed. At first, we demonstrate that DMC (2), derived from 1 by chlorination, can be used not only as a dehydrating agent, but also for the construction of a representative range of heterocycles and that $\mathbf{2}$ can be also used in chlorination, oxidation, reduction, and rearrangement reactions. Secondly modified guanidines, easily prepared from DMC derivatives by reaction with amines, could be acted as potential chiral auxiliaries in asymmetric synthesis, in which reasonable asymmetric induction are observed in both catalytic and stoichiometric modes. Finally, unique aziridine formation reactions applicable to asymmetric synthesis from guanidinium ylides and aryl aldehydes is established. Further development of guanidine chemistry has been on progress in our laboratory.

\section{Acknowledgements}

We thank our laboratory members for their hard work on these studies, especially Dr Toshio Isobe for his great contribution on DMC chemistry leading to the new roads. These works were 
supported by a Grant-in-Aid for Scientific Research from the Ministry of Education, Culture, Sports, Science and Technology, Japan.

\section{References}

1. (a) Isobe, T.; Ishikawa, T. J. Org. Chem. 1999, 64, 6984. (b) Isobe, T.; Ishikawa, T. J. Org. Chem. 1999, 64, 6989. (c) Isobe, T; Ishikawa, T. J. Org. Chem. 1999, 64, 5832.

2. Koenig, H. B.; Wilfried, S.; Cologne, H. D.; Metzger, K. G.; Ger. Offen. DE 2104579, 1972; Chem. Abstr. 1972, 77, 140,048. Koenig, H.-B.; Schroeck, W.; Disselnkoetter, H.; Metzger, K. G. US 3959258, 1976; Chem. Abstr. 1976, 85, 160,079.

3. March, J. In Advanced Organic Chemistry, 3rd Ed. Wiley-Interscience: New York, 1985, 1081.

4. (a) Isobe, T.; Fukuda, K.; Ishikawa, T. J. Org. Chem. 2000, 65, 7770. (b) Isobe, T.; Fukuda, K.; Tokunaga, T.; Seki, H.; Yamaguchi, K.; Ishikawa, T. J. Org. Chem. 2000, 65, 7774. (c) Isobe, T.; Fukuda, K.; Yamaguchi, K.; Seki, H.; Tokunaga, T.; Ishikawa, T. J. Org. Chem. 2000, 65, 7779 .

5. Kitani, Y.; Kumamoto, T.; Isobe, T.; Fukuda, K.; Ishikawa, T. Adv. Synth. Cat. 2005, 347, 1653.

6. Ishikawa, T.; Isobe, T. Chem. Eur. J. 2002, 8, 552.

7. Ishikawa, T.; Araki, Y.; Kumamoto, T.; Seki, H.; Fukuda, K.; Isobe, T. J. Chem. Soc. Chem. Commun. 2001, 245.

8. Isobe, T.; Fukuda, K.; Araki, Y.; Ishikawa, T. J. Chem. Soc. Chem. Commun. 2001, 243.

9. Hada, K.; Watanabe, T.; Isobe, T.; Ishikawa, T. J. Am. Chem. Soc. 2001, 123, 7705.

10. Haga, T.; Ishikawa, T. Tetrahedron 2005, 61, 2857. 